\title{
Estimation of Different Performance Parameters of Slotted Microstrip Antennas with Air-Gap Using Neural Networks
}

\author{
Taimoor Khan and Asok De \\ Department of Electronics and Communication Engineering, National Institute of Technology, Patna 800005, India \\ Correspondence should be addressed to Taimoor Khan; ktaimoor@gmail.com
}

Received 28 January 2014; Accepted 6 March 2014; Published 27 March 2014

Academic Editors: H. L. Hartnagel, L.-F. Mao, and Y.-H. Wang

Copyright ( 2014 T. Khan and A. De. This is an open access article distributed under the Creative Commons Attribution License, which permits unrestricted use, distribution, and reproduction in any medium, provided the original work is properly cited.

\begin{abstract}
Over the past decade, artificial neural networks have emerged as fast computational medium for predicting different performance parameters of microstrip antennas due to their learning and generalization features. This paper illustrates a neural network model for instantly predicting the resonance frequencies, gains, directivities, antenna efficiencies, and radiation efficiencies for dual-frequency operation of slotted microstrip antennas with air-gap. The proposed neural model is valid for any arbitrary slotdimensions and inserted air-gap within their specified ranges. A prototype is fabricated using Roger's substrate and its performance is measured for validation. A very good agreement is achieved in simulated, predicted, and measured results.
\end{abstract}

\section{Introduction}

There are many situations of wireless communication where dual-frequency operation is required such as satellite communication, radar systems, and global positioning system (GPS). Microstrip antennas (MSAs), because of operating in dual-frequency mode, have eliminated two singlefrequency operated antennas in these applications [1]. Different researchers have proposed different techniques for obtaining dual resonance such as multilayered stacked patch $[2,3]$, slotted rectangular patch [4], square patch with notches [5], patch loaded with shorting posts [6] or varactor diodes [7], and rectangular patch fed by an inclined slot [8]. These methods [2-8] can roughly be categorized as analytical methods and numerical methods. The analytical methods provide a good spontaneous explanation for the operation of MSAs. These techniques are based on the physical assumptions for simplifying the radiation mechanism of the MSAs but are not suitable for many microstrip structures where the thickness of the substrate is not very thin. On the other hand, the numerical methods provide accurate results but only at the cost of using complex mathematical expressions in the form of integral equations. The choice of test functions and path integrations appear to be more critical without initial assumptions in the final stage of the numerical results. Also, these approaches require a new solution even for an infinitesimal alteration in the geometry. Thus, the requirement for having a new solution for every small alteration in the geometry as well as the problems associated with the thickness of the substrates in analytical methods leads to complexities and processing cost [9]. Recently, artificial neural networks (ANNs) models have acquired tremendous applications in the wireless communication due to their ability and adaptability to learn and generalization features [9-16]. The ANN model is trained using measured, calculated or simulated patterns. The aim of the training process is to minimize error between reference and actual outputs of the ANN model. Once the model is trained for a specified error, it returns the results for every infinitesimal changes in the geometry both for electrically thin and thick MSAs within a fraction of a second. Several neural models are available in the literature [13-16] for computing single performance parameter (resonance frequency or geometric dimensions) of the microstrip patch antennas, respectively. But having individual neural model for each performance parameter becomes sometimes unattractive to include in modern antenna computer-aided-design (CAD) programs. It has recently been overcome by introducing 
the concept of generalized neural approach for computing different performance parameters, simultaneously [17-20]. The resonance frequencies of rectangular, circular, and triangular MSAs have been computed [17-19] using generalized neural networks model. The resonance frequencies and the physical dimensions of the rectangular MSAs have been computed using generalized neural networks model [20]. Hence, the individual neural models [9-16] and generalized neural approaches [17-20] have been used only for resonance frequency and/or geometric dimensions of microstrip patch antennas. Few neural networks models [21-23] have also been proposed for designing the slotted microstrip antennas. But unfortunately, simultaneous computations of different performance parameters (i.e., resonance frequency, gain, directivity, antenna efficiency, and radiation efficiency) using neural networks model have been rarely attempted in the available literature [9-23] as these parameters are essentially required for antenna designers for synthesizing the MSAs. In the proposed work, the authors have extended their earlier works of generalized neural networks modeling [24-30] for predicting different performance parameters (i.e., resonance frequencies, gains, directivities, antenna efficiencies, and radiation efficiencies) of slotted microstrip antennas for dualfrequency operation.

This paper is organized as follows. Proposed geometry of slotted microstrip antenna is discussed in Section 2. Section 3 describes proposed neural networks modeling. Experimental results and discussion are illustrated in Section 4. A conclusion followed by the references is discussed in Section 5 .

\section{Proposed Microstrip Antenna}

The proposed geometry of slotted MSA is shown in Figure 1. A rectangular patch of dimensions $61 \times 56 \mathrm{~mm}^{2}$ is designed with RT-Duroid substrate RO3003 $\left(\varepsilon_{r}=3, h=0.762 \mathrm{~mm}\right.$ and $\tan \delta=0.0045)$ using method of moment $(M o M)$ based IE3D simulator [31]. Two resonating modes $\left(\mathrm{TM}_{10}\right.$ and $\left.\mathrm{TM}_{01}\right)$ are excited by a single probe for getting dual resonance. The performance of the patch antenna is further improved by inserting air-gap of " $t$ " thickness between the substrate sheet and the ground plane [32]. A cross-slot of dimensions, " $x_{1}$," " $y_{1}$," " $x_{2}$ ", and " $y_{2}$ " is inserted on the radiating surface of rectangular patch microstrip antenna as shown in Figure 1. For each resonance, different performance parameters like gain, directivity, antenna efficiency, and radiation efficiency are observed by varying the slot-dimensions $(1 \mathrm{~mm} \leq$ slotdimensions $\leq 50 \mathrm{~mm})$ and inserted air-gap $(1 \mathrm{~mm} \leq$ air-gap $\leq$ $10 \mathrm{~mm}$ ). Flexible analytical modeling for such a complicated situation is still a challenge in electromagnetic community [1]. To tackle such problems there is no way except using the neural networks for fast estimation of the performance parameters for each value of slot-dimensions $(1 \mathrm{~mm} \leq$ slotdimensions $\leq 50 \mathrm{~mm})$ and inserted air-gap $(1 \mathrm{~mm} \leq$ airgap $\leq 10 \mathrm{~mm}$ ) for the geometry shown in Figure 1. The artificial neural networks modeling for the proposed problem is discussed in Section 3.

\section{Proposed Networks Modeling}

In today's highly integrated world, when solutions to problems are cross-disciplinary in nature, neural networks promise to become a powerful means for obtaining solutions to problems quickly and accurately. Artificial neural networks (ANNs) are massively distributed parallel processors that have natural propensity for storing experiential knowledge during training and making it available for use during testing. ANN models resemble the brain since knowledge is acquired by the network through a learning process, and inter-neuron connection strengths are used to store the knowledge [9]. Neural networks learn (or train) by known examples of a problem to acquire knowledge about it. Once the neural network is successfully trained, it can be put to effective use in solving "unknown" or "untrained" instances of the problem [9]. Multilayered perceptron (MLP) and radial basis function (RBF) neural networks have primarily been used in computing different performance parameters of the MSAs [9-30]. A radial basis function (RBF) neural network consists of three-layers feed-forward neural networks in which each layer is having entirely different roles [18, 19]. As far as learning (or training) of the neural networks is concerned, the RBF neural networks are much faster than the MLP neural networks. It is so because the learning process in RBF neural networks has two stages and both the stages are made more efficient by using appropriate learning algorithms [28]. There are three common steps followed during training of a neural networks model. Firstly, the training patterns are generated, then the structural configuration of hidden layer neurons is selected in the second step and finally, in the third step the weights are optimized by using training algorithm. For the first step, total 1350 patterns (1000-training + 350-testing patterns) are generated by varying the slot dimensions $(1 \mathrm{~mm}$ $\left.\leq x_{1}, y_{1}, x_{2}, y_{2} \leq 50 \mathrm{~mm}\right)$ and air-gap $(1 \mathrm{~mm} \leq t \leq 10 \mathrm{~mm})$ for the geometry shown in Figure 1. The structural configuration and training strategy of the proposed neural model are being discussed here.

To build a neural-network-based application, training stage is one of the more critical phases of the neural networks. Methods created from the field of optimization theory have played an important role in developing training algorithms for neural networks. Training a neural network model basically consists of adjusting weights with the help of a training algorithm. Before doing training, the generated patterns are normalized between +0.1 and +0.9 using MATLAB software [33]. The training performance of the proposed neural model is observed by varying the number of neurons in the hidden layer and after many trials, it is optimized with forty nine neurons for the best performance. Further, the training performance of the model is observed with seven different training algorithms, respectively [3335]. These are mentioned as BFGS quasi-Newton (BFG), Bayesian regulation (BR), scaled conjugate-gradient (SCG), Powell-Beale conjugate gradient (CGB), conjugate gradient with Fletcher-Peeves (CGF), one step secant (OSS), and Levenberg-Marquardt (LM).

A RBF neural network model for computing different performance parameters is illustrated in Figure 2 in 


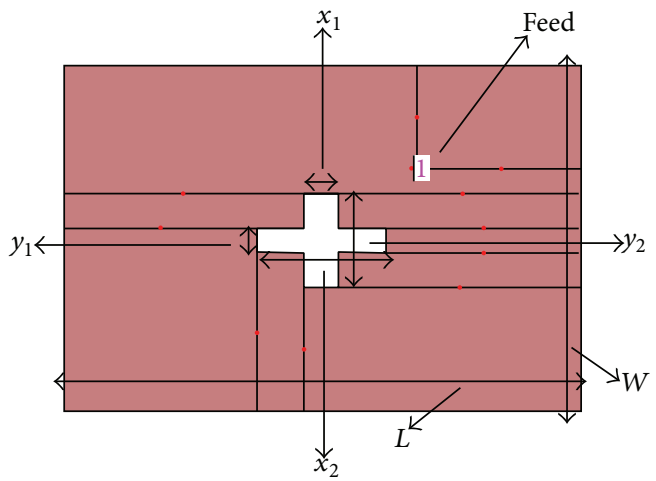

(a) Top view

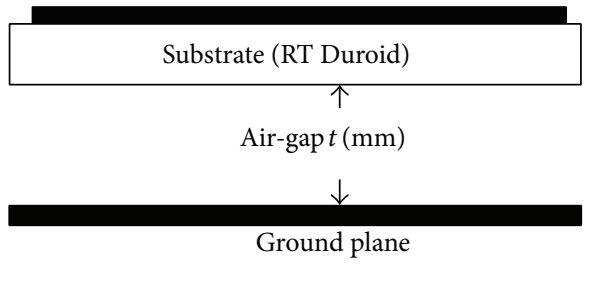

(b) Side view

FIGURE 1: Geometry of proposed antenna.

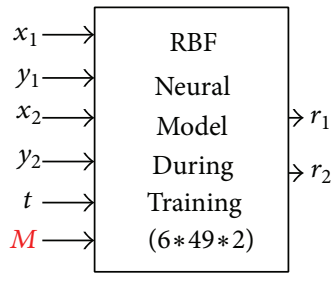

(a)

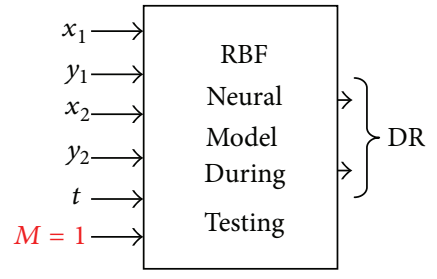

(b)

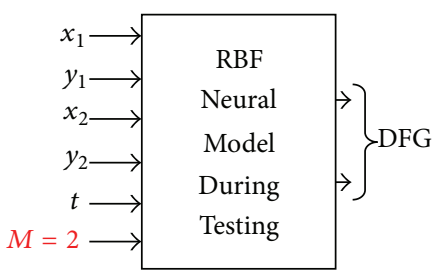

(c)

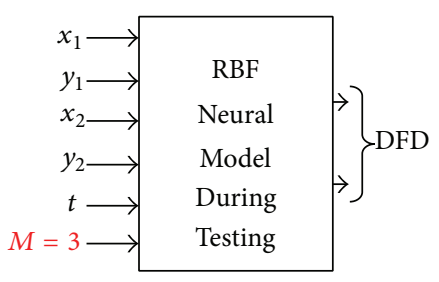

(d)

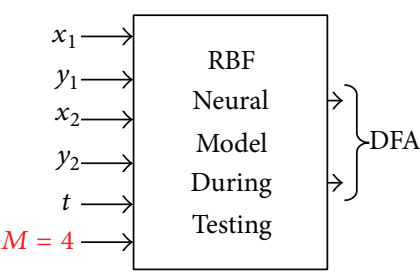

(e)

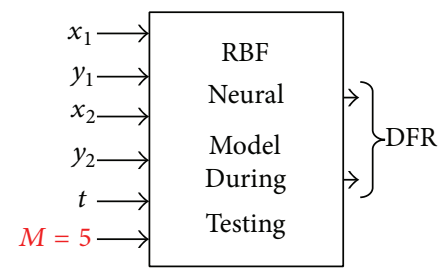

(f)

Figure 2: Proposed RBF neural model.

which input layer is having six-dimensional excitation matrix $\left[\begin{array}{llllll}x_{1} & y_{1} & x_{2} & y_{2} & t & M\end{array}\right]$ and output layer two-dimensional response matrix $\left[\begin{array}{ll}r_{1} & r_{2}\end{array}\right]$. Here variables " $x_{1}$," " $y_{1}$, " " $x_{2}$," and " $y_{2}$ " represent the dimensions of the cross-slot and " $t$ " indicates the inserted air-gap between the ground plane and the substrate (see Figure 1). As a common neural network model is being created for predicting different performance parameters of slotted MSAs for each value of slot-dimension and inserted air-gap, hence it becomes necessary to distinguish these performance parameters. For this purpose, an arbitrary parameter " $M$ " is included in the input patterns during training of the neural model. The parameter, " $M$," is treated here as mode control where $M=1,2,3,4$, and 5 corresponds to dual-resonance (DR), dual-frequency gains (DFG), dual-frequency directivities (DFD), dual-frequencies antenna efficiencies (DFA), and dual-frequencies radiation efficiency (DFR), respectively. Thus six-dimensional input pattern $[x] \rightarrow\left[\begin{array}{llllll}x_{1} & y_{1} & x_{2} & y_{2} & t & M\end{array}\right]$ and two-dimensional output pattern $[r] \rightarrow\left[\begin{array}{ll}r_{1} & r_{2}\end{array}\right]$ are used during training as well as testing of the proposed RBF neural networks model.
The block diagram used for training the proposed neural networks model is shown in Figure 2(a) whereas computations of different performance parameters during testing of the neural model are shown in Figures 2(b)-2(f).

The structural configuration for the proposed RBF neural model shown in Figure $2(\mathrm{a})$ is mentioned as $6 * 49 * 2$. For an applied input pattern, some random values between 0 and 1 are assigned to the weights and the output of the model is computed corresponding to that input pattern. The mean square error (MSE) between calculated and simulated results is then computed and all the weights are updated accordingly using a training algorithm. In the proposed neural modeling scheme seven different training algorithms, BFG, BR, SCG, CGB, CGF, OSS, and LM, are used [33-35]. This updating process is carried out after presenting each set of input pattern until the calculated accuracy of the neural model is deemed satisfactory. This updating process is completed by considering the training parameters: $\mathrm{MSE}=3.25 \times 10^{-6}$, learning rate $(\eta)=0.124$, momentum coefficient $(\mu)=0.432$, spread value $(\sigma)=0.469$, dimensionality of input pattern $(m)=6$, 
dimensionality of output pattern $(n)=2$, total number of pattern $(P)=1350$, number of training patterns $(M)=1000$, and number of testing pattern $(N)=350$. After optimizing the weights, the testing algorithm is implemented for the remaining 350 samples which were not included in 1000 training samples of the RBF model. For this implementation the initial weights are replaced by the optimized weights. The trained neural model now returns the response within a fraction of a second for any arbitrary values of crossslot dimensions $(1 \mathrm{~mm} \leq$ slot-dimensions $\leq 50 \mathrm{~mm})$ and airgap $(1 \mathrm{~mm} \leq$ air-gap $\leq 10 \mathrm{~mm})$. Computations of different performance parameters during testing of the neural model are illustrated in Figures 2(a)-2(f).

\section{Experimental Validation}

The neural networks modeling scheme is discussed in Section 2 for instantly predicting the desired performance parameters of the slotted microstrip antennas with inserted air-gap for dual-frequency operation. The training performance of the proposed neural model is observed with seven different algorithms, BFG, BR, SCG, CGB, CGF, OSS, and LM, respectively. In the present work, only LevenbergMarquardt (LM) backpropagation is proved to be the fastest converging training algorithm as it requires only $1489 \mathrm{sec}$. for training which is lower than that of other training algorithms. Further, for the proposed problem, this algorithm is also observed as more accurate than that of other algorithms.

The trained neural model returns the response for every infinitesimal change in the slot-dimension and air-gap within their specified ranges. It is tested for 350 samples of slotdimension and air-gap. The percentage error in computing the performance parameters for the first resonance $\left(T M_{10}\right.$ mode) is calculated. Thus, the average discrepancies between simulated and predicted values are observed as $1.92 \%, 0.9992 \%, 1.0937 \%, 2.9007 \%$, and $2.9490 \%$ in resonance frequencies, gains, directivities, antenna efficiencies, and radiation efficiencies, respectively. A graph is plotted in Figure 3 only for 50 values corresponding to first resonance $\left(T M_{10}\right.$ mode $)$. It is clear from Figure 3 that most of the predicted outcomes are in very good agreement with their simulated counterparts and only few are far off.

For validating the proposed work, a prototype is fabricated using RT-Duroid substrate $\mathrm{RO} 3003\left(\varepsilon_{r}=3\right.$ and $h=0.762 \mathrm{~mm}$ ). The patch of dimensions $61 \mathrm{~mm} \times 56 \mathrm{~mm}$ is etched on upper side of the substrate whereas an air-gap of $5.1 \mathrm{~mm}$ is stacked using Teflon rods between substrate and ground plane of dimensions $200 \times 175 \mathrm{~mm}^{2}$. The S-parameters of the fabricated prototype are measured using Agilent N5230A network analyzer and the setup used for this measurement is shown in Figure 4. The prototype is excited by probe feed which guides the electromagnetic waves to the feed point. The energy of electromagnetic waves then spreads out into the region under the slotted patch, some of which cross the boundary of the slotted patch and radiate into the half-space above the patch. An SMA connector with $6.8 \mathrm{~mm}$ long pin ( $1 \mathrm{~mm}$ for ground plane $+5.1 \mathrm{~mm}$ for airgap $+0.762 \mathrm{~mm}$ for substrate) is used for RF connection

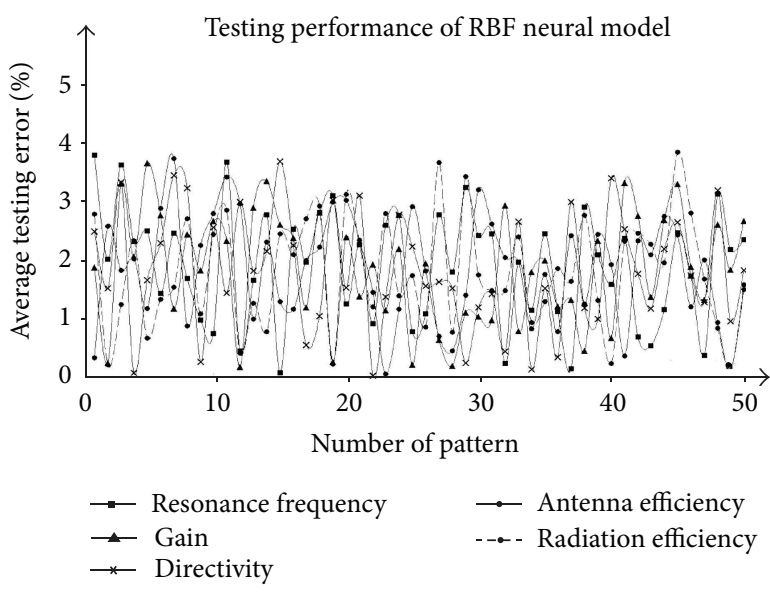

FIGURE 3: Comparison of error in testing data points.

TABLE 1: Comparison of results.

\begin{tabular}{lccc}
\hline Slot dimensions & \multicolumn{3}{c}{ Dual resonance $(\mathrm{GHz})$} \\
$(\mathrm{mm})$ & Simulated & Predicted & Measured \\
\hline$x_{1}=38.0, y_{1}=1.5$, & 1.7630 and & 1.7574 and & 1.7800 and \\
$x_{2}=1.0$ and $y_{2}=41.0$ & 1.9140 & 1.9136 & 1.9250 \\
\hline
\end{tabular}

between the ground plane and the slotted patch. Table 1 shows the measured results of the fabricated prototype. A good convergence among the simulated, calculated, and measured results confirms that the Taflon rods do not affect the antenna performance.

The performance parameters of the original geometry (without cross-slot) and cross-slotted geometry are studied and an improvement by inserting the cross-slot in the fabricated prototype is summarized in Table 2 . In the fabricated prototype, by inserting a cross-slot, the excited surface current path lengthens, increasing the antenna length and hence decreasing the resonance frequency. For the case of slotted patch, both the resonance frequencies $(1.763 \mathrm{GHz}$ and $1.914 \mathrm{GHz}$ ) are lowered by $\sim 13 \%$ as compared to those $(2.0256 \mathrm{GHz}$ and $2.1970 \mathrm{GHz})$ of the simple rectangular patch without a cross-slot. This reduction in the frequencies can result in a patch size reduction of $\sim 35 \%$ for a given dualfrequency design. Hence a good rank of compactness is achieved in the fabricated prototype. Also, the ratio $\left(f_{2} / f_{1}\right)$ of two frequencies $(1.914 / 1.763=1.0857$ and $2.1970 / 2.0256$ $=1.0846)$ is slightly affected by inserting the cross-slot and mainly determined by the aspect ratio $(1.89=61 / 56)$ of the patch, which makes the design simpler and easier to implement.

During simulation a bandwidth of $55.5 \mathrm{MHz}$ is achieved but it increases to $250 \mathrm{MHz}(1.99 \mathrm{GHz}-1.74 \mathrm{GHz})$ in fabricated prototype during measurement for $S_{11} \leq-10 \mathrm{~dB}$ (see Figure 4). It may be probably due to irregularity in fabricating the prototype and/or inserting the air-gap by using the Teflon rods. Further in the simulation, we do not consider the size and loss of the solder joint, which may cause this difference between the measured and simulated bandwidth. A very good impedance matching is also achieved by inserting 


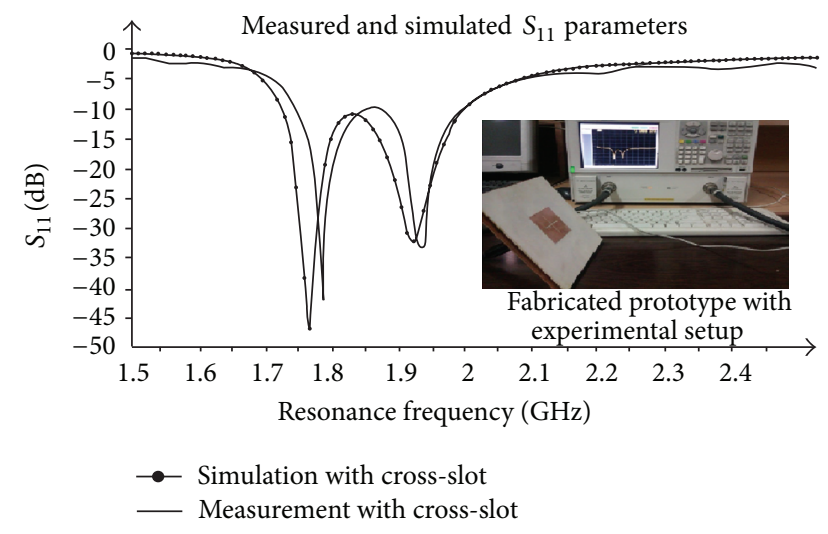

FIGURE 4: Comparison of measured and simulated S-parameters.

TABLE 2: Comparison of performance parameters.

\begin{tabular}{lcc}
\hline Parameters & Patch geometry & Slotted geometry \\
\hline DR $(\mathrm{GHz})$ & 2.0256 and 2.1970 & 1.7630 and 1.9140 \\
DFG $(\mathrm{dBi})$ & 8.2026 and 8.2129 & 8.9365 and 8.9387 \\
DFD $(\mathrm{dBi})$ & 9.1428 and 9.4193 & 9.1122 and 9.2079 \\
DFA $(\%)$ & 80.5329 and 85.3708 & 95.8065 and 93.9897 \\
DFR $(\%)$ & 90.2454 and 93.7718 & 96.3604 and 96.6599 \\
\hline
\end{tabular}

an air-gap of $5.1 \mathrm{~mm}$ (see Figure 4). The efficiencies (both antenna and radiation efficiencies) are improved because of negligible amount of dielectric loss in the air-layer. As gain of an antenna is defined as the product of directivity and radiation efficiency, hence the gain is also improved.

\section{Conclusion}

In this paper, a fast and accurate neural networks approach has been proposed for predicting different performance parameters of slotted microstrip antennas for dual-resonance. Using this approach, various radiation characteristics of the microstrip antennas for arbitrary slot-dimensions and airgap within their specified ranges have been computed. The proposed approach is capable of computing ten different parameters (dual-resonance, dual-frequency gains, dualfrequency directivities, dual-frequency antenna efficiencies, and dual-frequency radiation efficiencies), simultaneously as such an approach is rarely proposed in the literature for the best of our knowledge.

For validation a prototype has been fabricated and an air-gap has been stacked using Taflon rods between the ground plane and the substrate sheet. The performance of the fabricated prototype has been characterized using Agilent N5230A network analyzer. A very good convergence in the measured, calculated, and simulated results has been achieved.

In general, the neural model for computing ten different parameters may require ten different modules, whereas in the present work, only one model is fulfilling the requirement of the ten modules. Hence, this approach can be considered more generalized. This approach is simple and accurate that it can further be extended to a large number of computing parameters depending upon the required accuracy and structural configuration of the neural networks. Because of simplicity one can calculate the desired performance parameters for the given arbitrary values of slot-dimensions and air-gap within their specified ranges using a personal computer (PC) without having the fundamental knowledge of microstrip antennas. It takes only a fraction of a second in producing the required result on a computer system after being trained properly. As the approach has produced more accurate results in computing ten different parameters, hence it can be recommended to include in antenna computeraided-design (CAD) algorithms.

\section{Conflict of Interests}

The authors declare that there is no conflict of interests regarding the publication of this paper.

\section{References}

[1] I. J. Bahl and P. Bhartia, Microstrip Antennas, Artech House, Dedham, Mass, USA, 1980.

[2] J. S. Dahele, K.-F. Lee, and D. P. Wong, "Dual-frequency stacked annular-ring microstrip antennas," IEEE Transactions on Antennas and Propagation, vol. 35, no. 11, pp. 1281-1285, 1987.

[3] S. A. Long and M. D. Walton, "A dual-frequency stacked circular-disc antenna," IEEE Transactions on Antennas and Propagation, vol. 27, no. 2, pp. 270-273, 1979.

[4] S. Maci, G. B. Gentili, and G. Avitabile, "Single-layer dual frequency patch antenna," Electronics Letters, vol. 29, no. 16, pp. 1441-1443, 1993.

[5] H. Nakano and K. Vichien, "Dual-frequency square patch antenna with rectangular notch," Electronics Letters, vol. 25, no. 16, pp. 1067-1068, 1989.

[6] D. H. Schaubert, F. G. Farrar, A. Sindoris, and S. T. Hayes, "Microstrip antennas with frequency agility and polarization diversity," IEEE Transactions on Antennas and Propagation, vol. 29, no. 1, pp. 118-123, 1981.

[7] R. B. Waterhouse and N. V. Shuley, "Dual frequency microstrip rectangular patches," Electronics Letters, vol. 28, no. 7, pp. 606$607,1992$.

[8] Y. M. M. Antar, A. I. Ittipiboon, and A. K. Bhattacharyya, "A dual-frequency antenna using a single patch and an inclined slot," Microwave and Optical Technology Letters, vol. 8, no. 6, pp. 309-311, 1995.

[9] Q. J. Zhang and K. C. Gupta, Neural Networks for RF and Microwave Design, Artech House, Dedham, Mass, USA, 2000.

[10] P. M. Watson and K. C. Gupta, "EM-ANN models for microstrip vias and interconnects in dataset circuits," IEEE Transactions on Microwave Theory and Techniques, vol. 44, no. 12, pp. 24952503, 1996.

[11] P. M. Watson and K. C. Gupta, "Design and optimization of CPW circuits using EM-ANN models for CPW components," IEEE Transactions on Microwave Theory and Techniques, vol. 45, no. 12 , pp. 2515-2523, 1997. 
[12] P. M. Watson, K. C. Gupta, and R. L. Mahajan, "Development of knowledge based artificial neural network models for microwave components," in Proceedings of the IEEE MTT-S International Microwave Symposium Digest, vol. 1, pp. 9-12, Baltimore, Md, USA, June 1998.

[13] D. Karaboga, K. Guney, S. Sagiroglu, and M. Erler, "Neural computation of resonant frequency of electrically thin and thick rectangular microstrip antennas," IEE Proceedings H: Microwaves, Antennas and Propagation, vol. 146, no. 2, pp. 155-159, 1999.

[14] Ş. Sağiroğlu, K. Güney, and M. Erler, "Resonant frequency calculation for circular microstrip antennas using artificial neural networks," International Journal of RF and Microwave Computer-Aided Engineering, vol. 8, no. 3, pp. 270-277, 1998.

[15] Ş. Sağiroğlu and K. Güney, "Calculation of resonant frequency for an equilateral triangular microstrip antenna with the use of artificial neural networks," Microwave and Optical Technology Letters, vol. 14, no. 2, pp. 89-93, 1997.

[16] R. Gopalakrishnan and N. Gunasekaran, "Design of equilateral triangular microstrip antenna using artifical neural networks," in Proceedings of the IEEE International Workshop on Antenna Technology: Small Antennas and Novel Metamaterials (IWAT '05), pp. 246-249, March 2005.

[17] K. Güney, Ş. Sağiroğlu, and M. Erler, "Generalized neural method to determine resonant frequencies of various microstrip antennas," International Journal of RF and Microwave Computer-Aided Engineering, vol. 12, no. 1, pp. 131-139, 2002.

[18] K. Güney and N. Sarikaya, "A hybrid method based on combining artificial neural network and fuzzy inference system for simultaneous computation of resonant frequencies of rectangular, circular, and triangular microstrip antennas," IEEE Transactions on Antennas and Propagation, vol. 55, no. 3, pp. 659-668, 2007.

[19] K. Güney and N. Sarikaya, "Concurrent neuro-fuzzy systems for resonant frequency computation of rectangular, circular, and triangular microstrip antennas," Progress in Electromagnetics Research, vol. 84, pp. 253-277, 2008.

[20] N. Türker, F. Güneş, and T. Yildirim, "Artificial neural design of microstrip antennas," Turkish Journal of Electrical Engineering \& Computer Sciences, vol. 14, no. 3, pp. 445-453, 2006.

[21] D. K. Neog, S. S. Pattnaik, D. C. Panda, S. Devi, B. Khuntia, and M. Dutta, "Design of a wideband microstrip antenna and the use of artificial neural networks in parameter calculation," IEEE Antennas and Propagation Magazine, vol. 47, no. 3, pp. 60-65, 2005.

[22] V. V. Thakare and P. K. Singhal, "Bandwidth analysis by introducing slots in microstrip antenna design using ANN," Progress In Electromagnetics Research M, vol. 9, pp. 107-122, 2009.

[23] V. V. Thakare and P. Singhal, "Microstrip antenna design using artificial neural networks," International Journal of RF and Microwave Computer-Aided Engineering, vol. 20, no. 1, pp. 76-86, 2010.

[24] T. Khan and A. De, "Computation of different parameters of triangular patch microstrip antennas using a common neural model," International Journal of Microwave and Optical Technology, vol. 5, no. 4, pp. 219-224, 2010.

[25] T. Khan and A. De, "A common neural approach for computing different parameters of circular patch microstrip antennas," International Journal of Microwave and Optical Technology, vol. 6, no. 5, pp. 259-262, 2011.

[26] T. Khan and A. De, "Design of circular/triangular patch microstrip antennas using a single neural model," in Proceedings of the
IEEE Applied Electromagnetic Conference (AEMC '11), pp. 1-4, Kolkata, India, December 2011.

[27] T. Khan and A. De, "A generalized neural simulator for computing different parameters of circular/triangular microstrip antennas simultaneously," in Proceedings of the IEEE AsiaPacific Conference on Applied Electromagnetics (APACE '12), pp. 350-354, Melaka, Malaysia, December 2012.

[28] T. Khan and A. De, "A generalized neural method for simultaneous computation of resonant frequencies of rectangular, circular and triangular microstrip antennas," in Proceedings of the International Conference on Global Innovation Technology and Science (ICGITS '13), Saintgits College of Engineering, Kerala, India, April 2013.

[29] T. Khan and A. De, "Prediction of resonant frequencies of rectangular, circular and triangular microstrip antennas using a generalized RBF neural model," International Journal of Scientific and Engineering Research, vol. 4, no. 8, pp. 182-187, 2013.

[30] T. Khan and A. De, "A generalized ANN model for analyzing and synthesizing rectangular, circular and triangular microstrip antennas," Chinese Journal of Engineering, vol. 2013, Article ID 647191, 9 pages, 2013.

[31] IE3D Version 14.0, Zeland Software, Fremont, Calif, USA, 2007.

[32] J. S. Dahele and K. F. Lee, “Theory and experiment on microstrip antennas with airgaps," IEEE Proceedings H: Microwaves, Antennas and Propagation, vol. 132, no. 7, pp. 455-460, 1985.

[33] D. J. Higham and N. J. Higham, MATLAB Guide, SIAM, Philadelphia, Pa, USA, 2005.

[34] M. T. Hagan and M. B. Menhaj, "Training feedforward networks with the Marquardt algorithm," IEEE Transactions on Neural Networks, vol. 5, no. 6, pp. 989-993, 1994.

[35] S. Chen, C. F. N. Cowan, and P. M. Grant, "Orthogonal least squares learning algorithm for radial basis function networks," IEEE Transactions on Neural Networks, vol. 2, no. 2, pp. 302-309, 1991. 

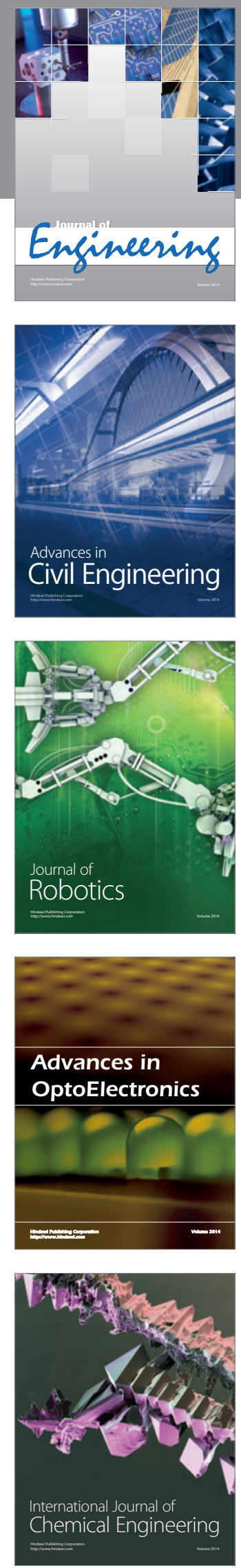

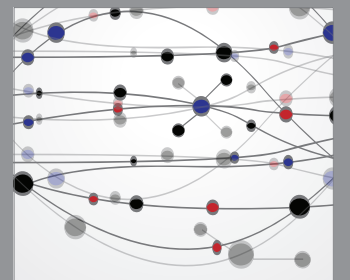

The Scientific World Journal
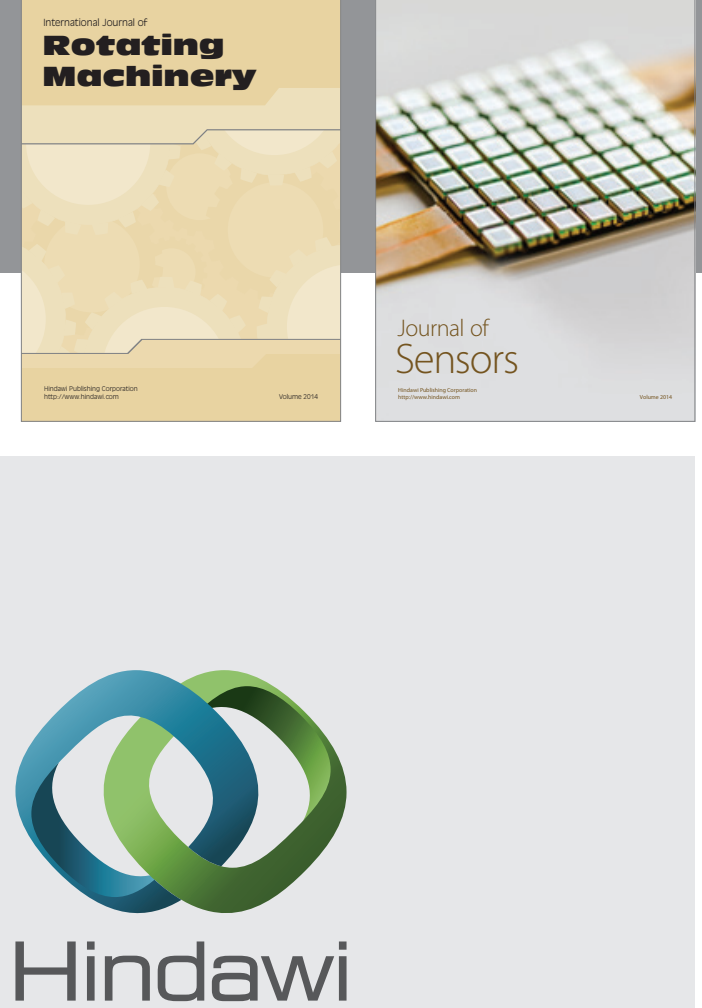

Submit your manuscripts at http://www.hindawi.com
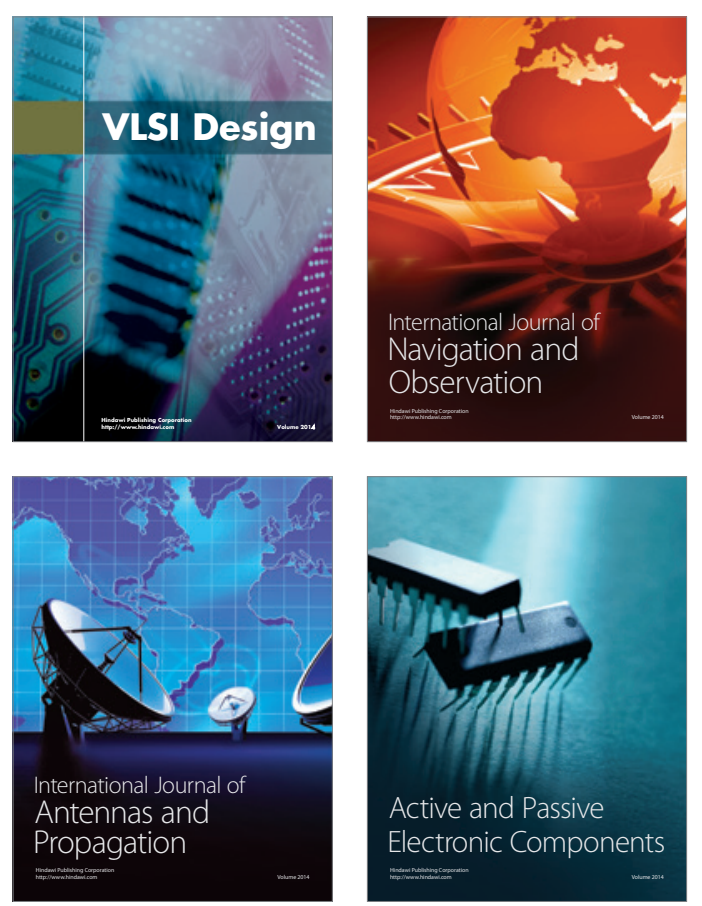
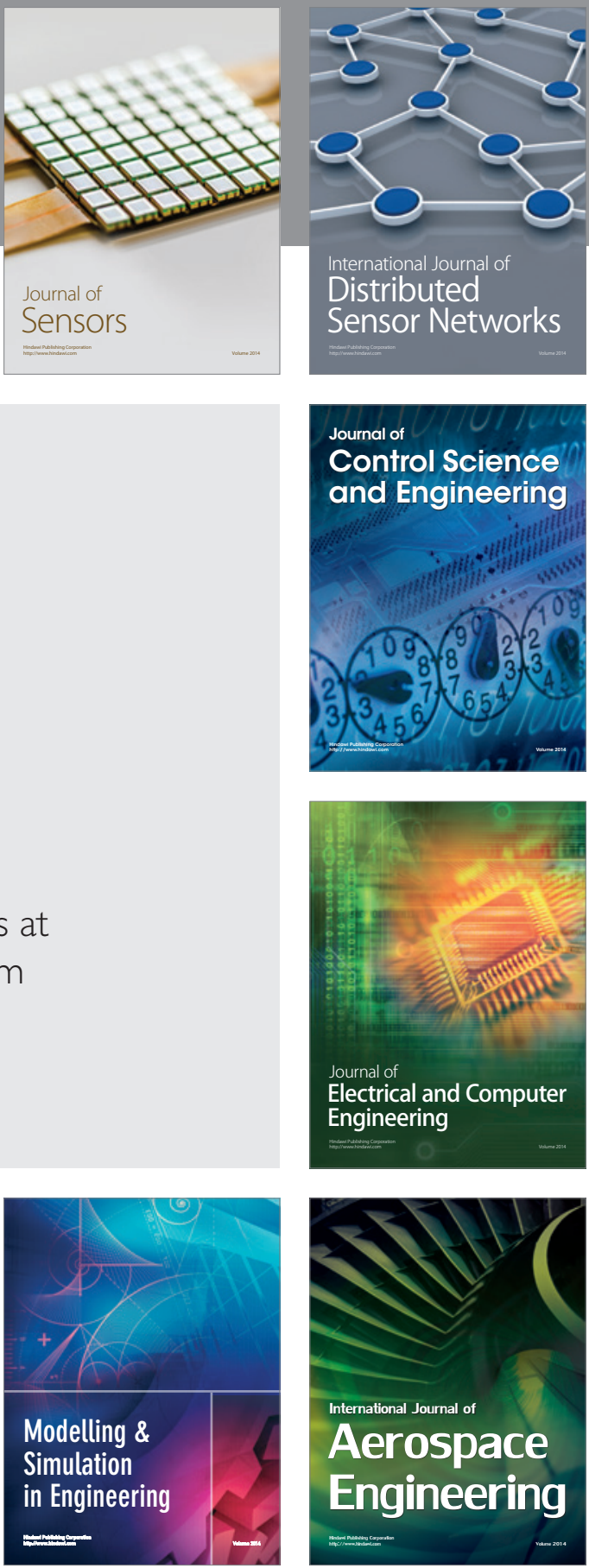

Journal of

Control Science

and Engineering
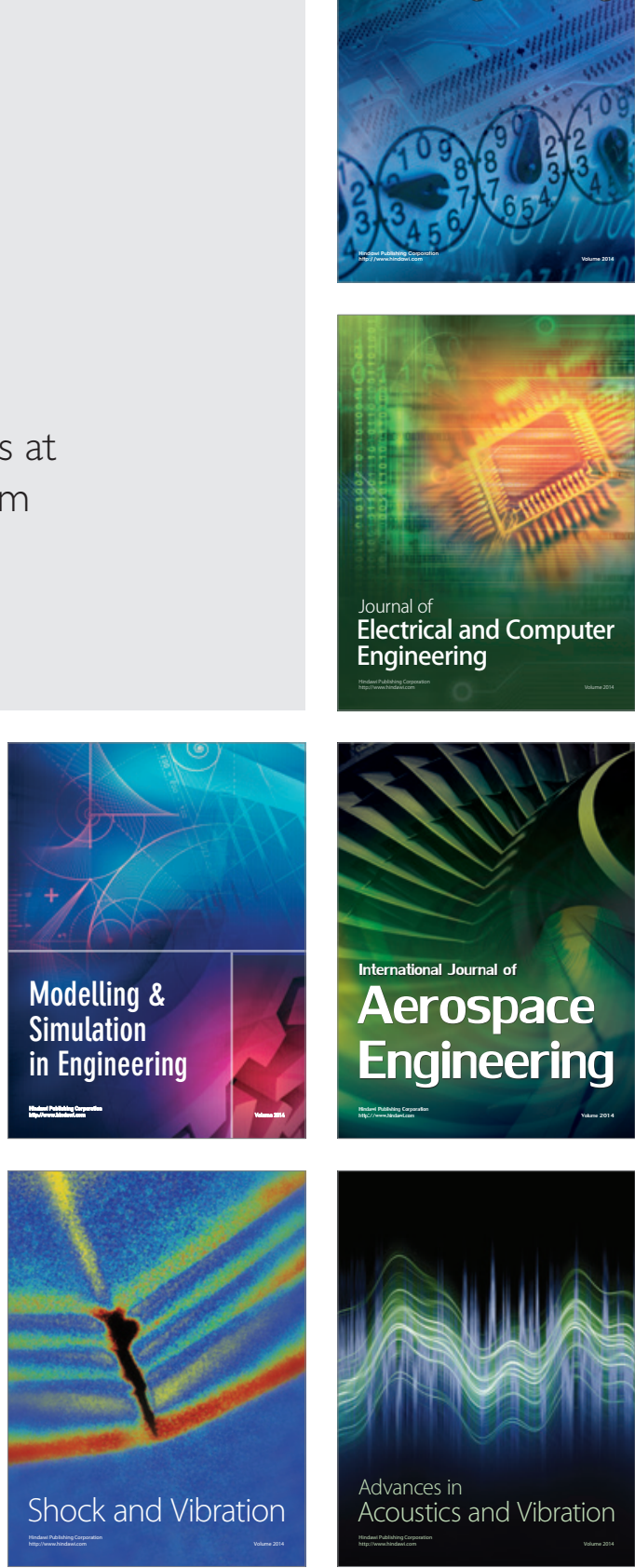